\title{
Initial experience with a new technique of endoscopic and ultrasonographic access for biopsy of para-aortic (station 6) mediastinal lymph nodes without traversing the aorta
}

\author{
Moishe Liberman, MD, PhD, André Duranceau, MD, Etienne Grunenwald, MD, Vicky Thiffault, RN, \\ Mohamed Khereba, MD, and Pasquale Ferraro, MD
}

\begin{abstract}
Objectives: All lymph node stations but the para-aortic are accessible by a combination of endoscopic ultrasound and endobronchial ultrasound. We recently described an echographic-endoscopic technique for the biopsy of para-aortic (station 6) lymph nodes without traversing the thoracic aorta. This study reviewed our initial experience with this new technique.
\end{abstract}

\begin{abstract}
Methods: This first-in-human evaluation of the biopsy of station 6 mediastinal lymph nodes with curvilinear endoscopic ultrasound without arterial puncture used a retrospective case series design to study 12 consecutive patients who underwent this new technique. Station 6 lymph nodes were approached with a long fine needle aspiration approach $(6-8 \mathrm{~cm})$ through the proximal esophagus. The needle was passed through the esophagus into the mediastinum just medial to the left subclavian artery. It was then directed toward the para-aortic location (6-8 cm trajectory) to reach and enter the para-aortic lymph nodes without piercing the aorta or great vessels.
\end{abstract}

Results: Successful cytologic diagnoses of station 6 lymph nodes were obtained in all cases (lymphocytes in all samples). No morbidity resulted from the procedure, nor was any observed at 30 days after the procedure. Patient anatomy may preclude safe access in certain situations.

Conclusions: Endoscopic ultrasound access of para-aortic (station 6) lymph nodes allows complete, minimally invasive mediastinal lymph node staging and diagnosis without traversal of the aorta. This technique, the final piece of the puzzle required for complete staging of the mediastinum with nonsurgical endoscopic techniques, is reproducible and safe. (J Thorac Cardiovasc Surg 2012;144:787-93)

Endoscopic ultrasound (EUS) and endobronchial ultrasound (EBUS) with transluminal biopsy of mediastinal lymph nodes have together revolutionized the diagnosis and staging of the mediastinum in patients with mediastinal lymph node metastases. Traditionally, access to mediastinal lymph nodes has been obtained by surgical mediastinoscopy, anterior mediastinotomy, video-assisted thoracoscopic surgery (VATS), or thoracotomy. ${ }^{1-8}$ With the advent of echographic-endoscopic technology, through both the esophagus and tracheobronchial tree, minimally invasive, transluminal, nonsurgical lymph node aspiration and biopsy for cytologic and histologic analysis is possible.

\footnotetext{
From the Division of Thoracic Surgery, Department of Surgery, CHUM Endoscopic Tracheobronchial and Oesophageal Center, University of Montréal, Montréal, Québec, Canada.

Disclosures: Authors have nothing to disclose with regard to commercial support.

Read at the 92nd Annual Meeting of The American Association for Thoracic Surgery, San Francisco, California, April 28-May 2, 2012.

Received for publication March 31, 2012; revisions received June 11, 2012; accepted for publication July 9, 2012.

Address for reprints: Moishe Liberman, MD, PhD, Division of Thoracic Surgery, Centre Hospitalier de l'Université de Montréal, 1560 rue Sherbrooke Est, 8e CD Pavillon Lachapelle, Bureau D-8051, Montréal, Québec, Canada H2L 4M1 (E-mail: moishe.liberman@umontreal.ca).

$0022-5223 / \$ 36.00$

Copyright (C) 2012 by The American Association for Thoracic Surgery

http://dx.doi.org/10.1016/j.jtcvs.2012.07.003
}

These techniques are rapidly replacing traditional surgical methods for lymph node biopsy.

All lymph node stations, except the para-aortic lymph node station, are directly accessible with a combination of EUS and EBUS (Table 1). ${ }^{9,10}$ Because of the anatomic constraints of the aortic arch, however, both EBUS and EUS have been thought to be incapable of accessing the level 6 (para-aortic) lymph node station without traversing the aorta (Figures 1 and 2). The station 6 nodes sit on the lateral portion of the aortic arch. ${ }^{11}$ Transbronchial ultrasound-guided biopsy with EBUS would require a needle path through the left main stem bronchus, through the medial and lateral walls of the aorta, and then into the lymph nodes. EUS access of these nodes was also thought to be impossible, requiring puncture of the pulmonary artery, aorta, or both to reach the para-aortic location. Although transaortic biopsy of mediastinal lymph nodes and lung masses has been previously described, ${ }^{12,13}$ it has a low likelihood of gaining widespread acceptance because of the theoretic risks associated with a transaortic puncture.

We have recently described an echographic-endoscopic technique for the biopsy of para-aortic (station 6) lymph nodes without traversing the thoracic aorta or pulmonary artery. ${ }^{14}$ This is the final frontier in complete minimally invasive, natural orifice mediastinal lymph node staging. 


\section{Abbreviations and Acronyms \\ $\mathrm{CT}=$ computed tomography \\ EBUS $=$ endobronchial ultrasound \\ EUS = endoscopic ultrasound \\ VATS $=$ video-assisted thoracoscopic surgery}

The purpose of this study is to review our initial experience and results with this new technique in a series of consecutive patients.

\section{MATERIALS AND METHODS}

This study consists of a retrospective case series. It is a review of the initial experience with a novel technique consisting of the biopsy of station 6 (para-aortic) mediastinal lymph nodes with curvilinear EUS without arterial puncture. All cases were drawn from a prospectively accrued thoracic surgery interventional endoscopy research database. The study reports the cases of 12 consecutive patients who underwent staging with this new technique.

All patients provided informed consent for echographic-endoscopic mediastinal lymph node staging with a combined EBUS-EUS approach before their procedures. The study was approved by the institutional review board at the Centre de Recherche du CHUM, University of Montreal. All patients underwent the procedure with either general anesthesia or local anesthesia with mild to heavy sedation. Anesthesia choice was based on endoscopist preference and was subjectively determined on the basis of patient clinical characteristics and computed tomographic (CT) scan criteria. Most patients underwent EBUS-EUS procedures under local anesthesia with mild to moderate sedation.

Because of the technical difficulty with the biopsy of station 6 lymph nodes and the risks related to the procedure, attempted biopsy of station 6 lymph nodes was only performed in a clinical situation in which there were either no other lymph node stations that were radiologically suspect according to $\mathrm{CT}$ scan $(<1 \mathrm{~cm}$ short axis) or according to positron emission tomography-CT (fludeoxyglucose nonavid) and in which the biopsy of station 6 was considered to have the potential to alter the management plan for the specific patient. Patients with ectatic left subclavian arteries, severe calcification of the aortic arch or left subclavian artery, inability to remain still during the procedure, lymph nodes too lateral or inferior on the aortic arch, or lymph nodes deemed by the endoscopist to be too small for safe biopsy did not have biopsy attempted with the described technique.

The first of these procedures was performed in December 2010, and the last procedure was performed in September 2011 (10 months). During the period covered by the study, 395 interventional thoracic endoscopic procedures and 274 combined EBUS-EUS procedures were performed at the CHUM Endoscopic Tracheobronchial and Oesophageal Center, Division of Thoracic Surgery, Centre Hospitalier de l'Université de Montréal. EUS access for station 6 was deemed necessary and attempted in only 12 of the 274 mediastinal staging procedures $(4.4 \%)$.

All patients underwent plain film chest radiography after their procedures to rule out mediastinal hematoma, pneumothorax, and hemothorax. Patients were followed up at 30 days after the procedure by either telephone interview or outpatient clinic visit.

\section{Technique}

Differentiation between stations 5 and 6 was made on the basis of preprocedural CT scan images and the real-time EUS imaging during the procedure. On EUS imaging, however, one is able to identify many more lymph nodes than on CT scan because of the echogenic characteristics of most lymph nodes. Any lymph node that was found on EUS to be lateral
TABLE 1. Endoscopic ultrasound and endobronchial ultrasound accessibility for mediastinal lymph node stations

\begin{tabular}{llclc}
\hline & & \multicolumn{3}{c}{ Accessible } \\
\cline { 3 - 5 } LN station & \multicolumn{1}{c}{ Description } & EBUS & EUS & $\begin{array}{c}\text { EBUS } \\
\text { and EUS }\end{array}$ \\
\hline 1 & Highest mediastinal & Yes & No & No \\
2R & Right upper paratracheal & Yes & Difficult & Yes \\
2L & Left upper paratracheal & Yes & Yes & Yes \\
3A & Presvascular & Yes & No & No \\
3P & Retrotracheal & Yes & Yes & Yes \\
4R & Right lower paratracheal & Yes & Difficult & Yes \\
4L & Left lower paratracheal & Yes & Yes & Yes \\
5 & Subaortic (aortopulmonary & No & Yes & No \\
& window) & & & \\
6 & Para-aortic & No & New & No \\
7 & Subcarinal & Yes & Yes & Yes \\
8R, 8L & Paraesophageal & No & Yes & No \\
9R, 9L & Pulmonary ligament & No & Yes & No \\
10R, 10L & Hilar & Yes & No & No \\
11R, 11L & Interlobar & Yes & No & No \\
12R, 12L & Lobar & Yes & No & No \\
\hline
\end{tabular}

Lymph node stations are designated according to the International Association for the Study of Lung Cancer scheme. EBUS, Endobronchial ultrasound; EUS, endoscopic ultrasound; $L N$, lymph node.

to the aortic arch (para-aortic) was identified as a station 6 lymph node, whereas any node that lay between the aorta and the left main pulmonary artery was identified as a station 5 lymph node.

Station 6 lymph nodes are visualized during convex EUS by locating the aortic arch on the left lateral side of the mediastinum, approximately 1 to 2 $\mathrm{cm}$ above the upper edge of the left main stem bronchus, and scanning in a radial manner while looking through the aortic arch (Figures 3 and 4). During EUS access of para-aortic (station 6) lymph nodes, lymph nodes were approached through the proximal esophagus (20-25 cm from the incisors). An EUS fine-needle aspiration needle was passed through the esophageal wall into the mediastinum immediately medial to the left subclavian artery and then directed toward the para-aortic location. The needle was passed through the esophagus into the mediastinum immediately next to the left subclavian artery, 4 to $5 \mathrm{~cm}$ distal to its takeoff from the aortic arch. It was then directed toward the para-aortic location with a 6- to 8 -cm trajectory to reach and enter any para-aortic lymph nodes without piercing the aorta or great vessels (Figures 5 and 6). The lymph node was not visualized when passing the needle out into the mediastinum, and a mental image of where it is located therefore had to be kept in the endoscopist's head as the scope was slowly withdrawn above the aortic arch.

All procedures in this study were performed with a curvilinear EUS scope (Olympus GF-UC140P-AL5; Olympus Canada Inc, Richmond Hill, Ontario, Canada; and Aloka Alpha 10 Processor, Hitachi-Aloka Medical, Tokyo, Japan) and a 22-guage EUS fine-needle aspiration needle (Cook EchoTip Ultra EUS needle; Cook Medical, Bloomington, Ind). At minimum, 2 passes per lymph node station were performed. Cytologic slides as well as a histologic cell block were prepared for analysis by the surgical team, without on-site cytologic technicians or cytopathologists.

\section{RESULTS}

Diagnosis, biopsy result, and echographic lymph node characteristics are described in Table 2. Successful cytologic diagnoses of station 6 lymph nodes were obtained in 


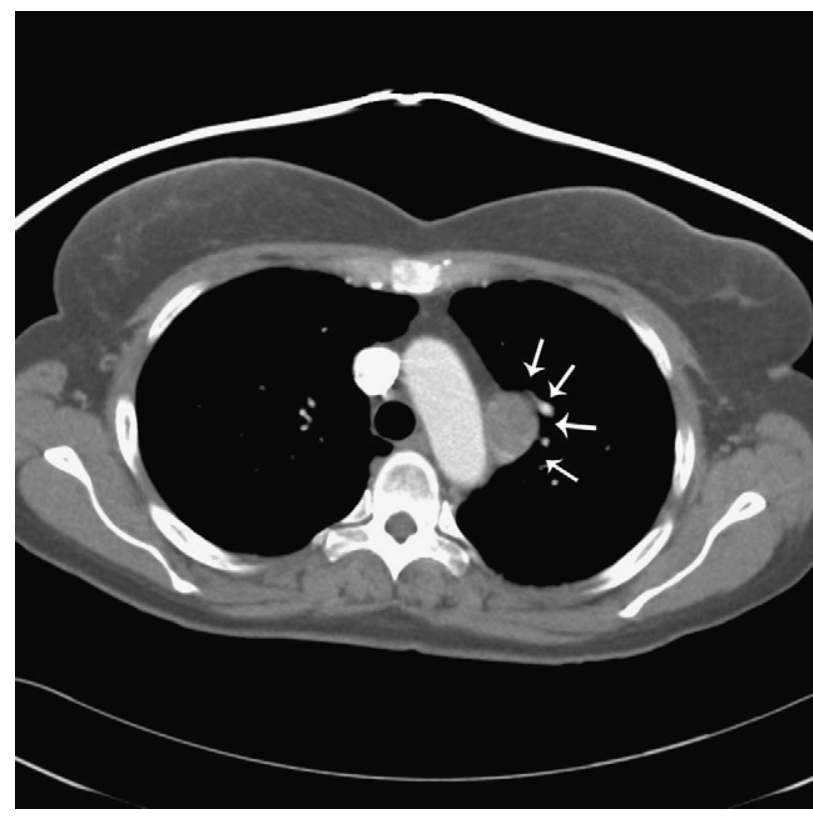

FIGURE 1. Axial computed tomographic scan image demonstrating paraaortic lymphadenopathy in the station 6 location. Enlarged lymph nodes in the para-aortic position (station 6) are marked with arrows.

all 12 consecutive patients (lymphocytes in all samples). There were no insufficient samples. Two patients considered eligible for EUS biopsy of station 6 lymph nodes according to clinical and radiologic criteria were excluded from the series because of anatomic constraints (ectatic left subclavian artery, heavily calcified aorta).

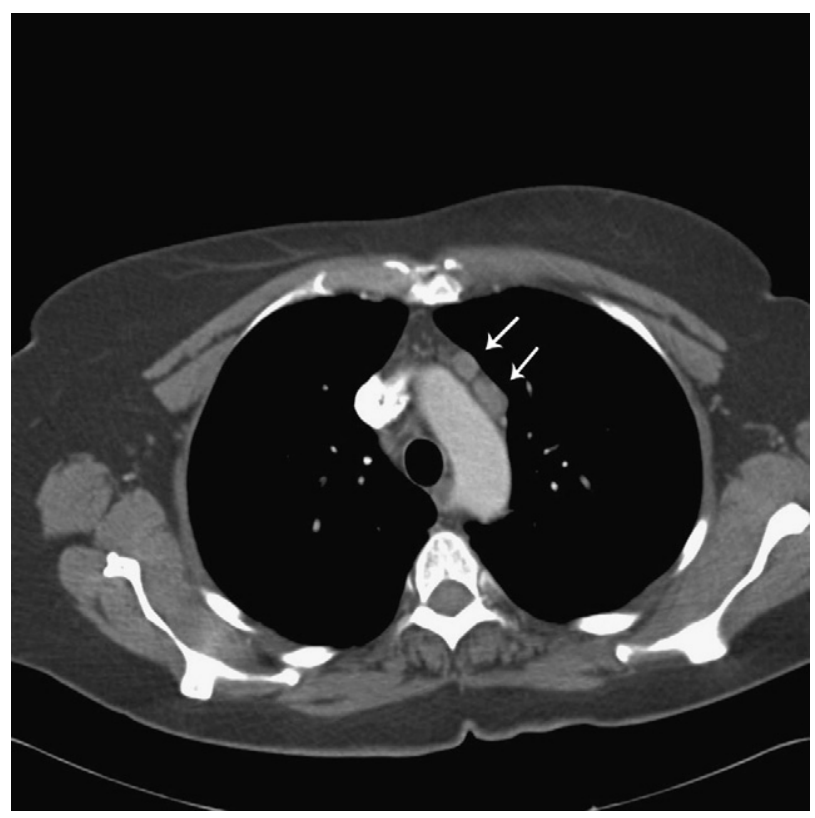

FIGURE 2. Axial computed tomographic scan image demonstrating paraaortic lymph nodes in the station 6 location. Lymph nodes in the para-aortic position (station 6) are marked with arrows.

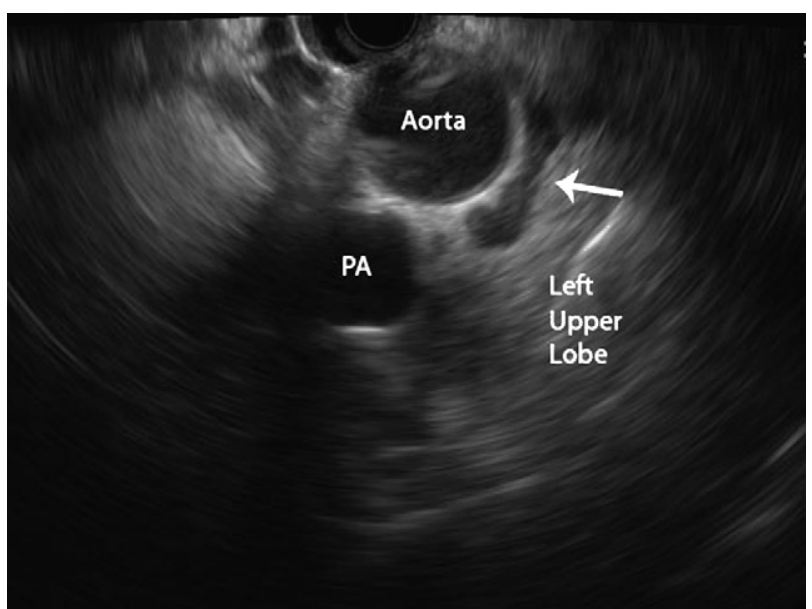

FIGURE 3. Curvilinear endoscopic ultrasound image of a lymph node in the station 6 position. The station 6 lymph node is marked with an arrow. $P A$, Left pulmonary artery.

One patient in this study (patient 11; Table 2) underwent surgical confirmation of the station 6 lymph node. This patient had a large $(8 \times 8 \times 6 \mathrm{~cm})$ left upper lobe and hilar adenocarcinoma. She underwent complete minimally invasive mediastinal lymph node staging with a combined EBUS-EUS procedure. All lymph nodes biopsied (11L, $4 \mathrm{R}, 4 \mathrm{~L}, 5$, and 7), including the station 6 lymph node, yielded negative results on cytologic analysis. The station 6 lymph node was measured by EUS to be $1.0 \times 0.9 \mathrm{~cm}$; it had low-echogenicity, nonsharp borders and was oval in shape. The patient subsequently underwent a left VATS pneumonectomy on a separate occasion. Final pathologic study of the thoracoscopic mediastinal lymph node dissection at that time confirmed that all lymph nodes, including the station 6 lymph nodes, were free of malignancy.

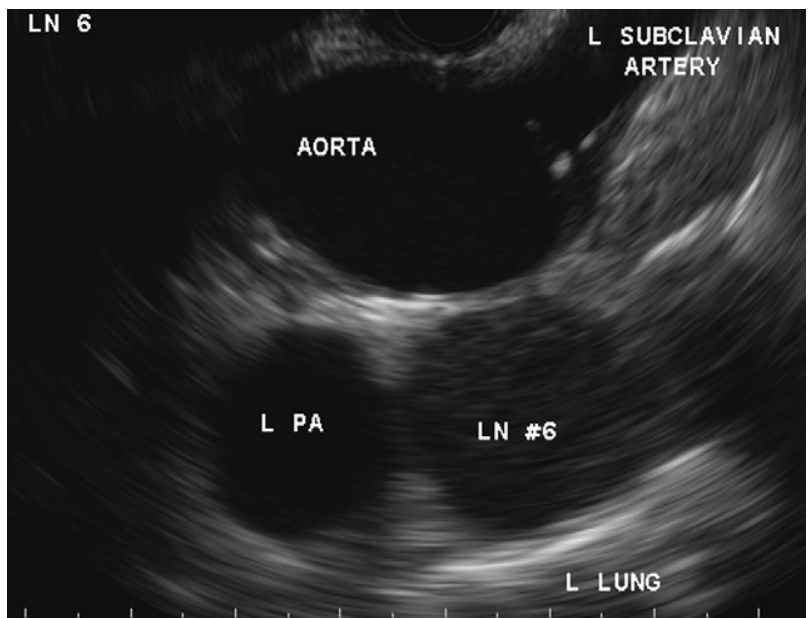

FIGURE 4. Curvilinear endoscopic ultrasound image of an enlarged lymph node $(L N)$ in the station 6 position. The station 6 lymph node is labeled $L N \# 6$. $L$, Left; $P A$, pulmonary artery. 


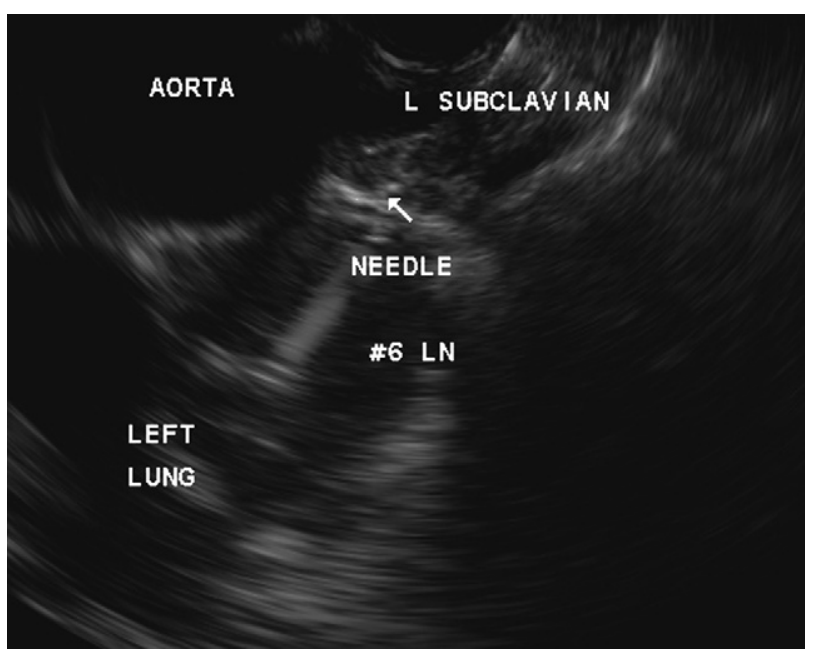

FIGURE 5. Curvilinear endoscopic ultrasound image of a lymph node $(L N)$ in the station 6 position during fine-needle aspiration biopsy. The needle is marked with an arrow. $L$, Left.

No other patient in this study underwent surgical confirmation of station 6 lymph node status. This is because in this cohort either station 6 lymph nodes were positive and the patients were ineligible for surgical resection or neoadjuvant therapy followed by surgical resection, or the nodes were negative but the patients had extrathoracic malignancy (colon cancer or anal cancer).

No morbidity resulted from the procedure. No occurrences of pneumothorax, pneumomediastinum, hemothorax, or mediastinal hematoma were observed on postprocedural chest radiographs. No morbidity related to the EUS procedure was observed in any patient at 30-day follow-up.

\section{DISCUSSION}

Lymph nodes within the mediastinum have traditionally been staged with surgical lymph node biopsy by means of cervical mediastinoscopy, anterior mediastinotomy and mediastinoscopy, thoracoscopy, or thoracotomy. ${ }^{1-8}$ EUS and EBUS as minimally invasive modalities for staging the mediastinum without surgery are quickly replacing traditional surgical techniques. ${ }^{9,10}$ Before the introduction of the described technique, the combination of EBUS and EUS allowed almost complete, minimally invasive, endoscopic mediastinal lymph node staging and diagnosis for all mediastinal lymph node stations ${ }^{11,15}$ except the para-aortic lymph node station (station 6). ${ }^{16,17}$ Combined EBUS-EUS allows a "complete medical mediastinoscopy," 18 which allows access not only to all mediastinal lymph node stations but also to the bilateral adrenal glands, the celiac axis lymph nodes, and the liver. EBUS and EUS are complementary with one another ${ }^{19,20}$ and can even complement surgical $^{21,22}$ and radiologic ${ }^{23}$ staging. Table 1 outlines techniques for minimally invasive access to mediastinal lymph nodes by EBUS and EUS.

This report describes the successful application of a new technique for access to the para-aortic lymph node stations without the added risk of transaortic puncture. The technique is technically demanding and requires not only a great deal of precision but also the cooperation of the patient in using deep sedation or general anesthesia.

Although no morbidity was observed in this series, potential dangers to the procedure are related to the long transmediastinal trajectory, with risks of bleeding, pneumothorax, lung laceration, esophageal laceration with fistula formation, and mediastinal abscess. Furthermore, the
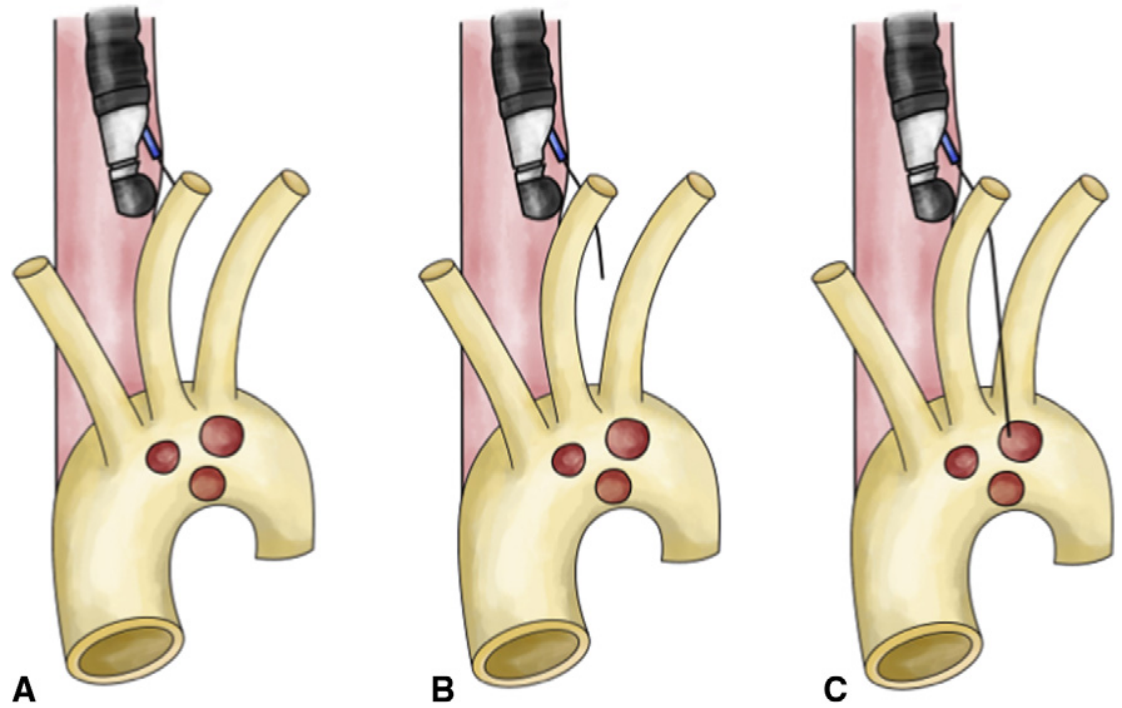

FIGURE 6. Schematic drawing depicting the technique of endoscopic ultrasonographic access to para-aortic mediastinal lymph nodes. Lymph nodes are approached by a long approach through the proximal esophagus. The needle is passed immediately medial to the left subclavian artery (A). It then is passed 4 to $5 \mathrm{~cm}$ distal to its takeoff from the aortic arch and is then turned inferiorly and laterally once past the artery (B) to approach the station 6 lymph nodes (C). 
TABLE 2. Endoscopic ultrasound station 6 lymph node biopsy: Patient characteristics

\begin{tabular}{|c|c|c|c|c|c|c|c|}
\hline Patient & Diagnosis & LNs in position 6 & Size of LN biopsied $(\mathrm{cm})$ & LN echogenicity & LN borders & LN shape & EUS LN 6 result \\
\hline 1 & Esophageal cancer & 1 & $1.2 \times 0.9$ & Medium & Hazy & Polypoid & Positive \\
\hline 2 & Cervical cancer & 1 & $1.6 \times 0.9$ & Medium & Sharp & Triangular & Positive \\
\hline 3 & NSCLC & 1 & $2.1 \times 1.6$ & Low & Sharp & Round & Positive \\
\hline 4 & SCLC & 1 & $2.6 \times 2.1$ & High & Sharp & Round & Positive \\
\hline 5 & NSCLC & 1 & $0.8 \times 0.9$ & High & Sharp & Round & Positive \\
\hline 6 & Breast cancer & 1 & $1.9 \times 1.4$ & High & Hazy & Polypoid & Positive \\
\hline 7 & NSCLC & 2 & $1.7 \times 1.6$ & Low & Sharp & Round & Positive \\
\hline 8 & Colon cancer & 1 & $1.1 \times 1.1$ & Medium & Hazy & Polypoid & Negative \\
\hline 9 & Anal cancer & 3 & $1.4 \times 1.0$ & Medium & Sharp & Round & Negative \\
\hline 10 & NSCLC & 1 & $3.7 \times 2.6$ & High & Sharp & Round & Positive \\
\hline 11 & NSCLC & 1 & $1.0 \times 0.9$ & Low & Hazy & Oval & Negative \\
\hline 12 & NSCLC & 1 & $1.7 \times 1.3$ & Medium & Hazy & Round & Negative \\
\hline
\end{tabular}

EUS, Endoscopic ultrasound; $L N$, lymph node; $N S C L C$, non-small cell lung cancer; $S C L C$, small cell lung cancer.

proximity of the needle to the left subclavian artery is associated with risks of arterial puncture, laceration, and pseudoaneurysm formation.

Patient anatomy may preclude this procedure. Anatomic factors that may make this procedure difficult or impossible include enlarged diameter of the transverse or ascending aortic arch, early takeoff of the left subclavian artery, aneurysmal dilation of the left subclavian artery, heavily calcified aorta, and lymph node location very caudad on the descending aortic arch. There were 274 patients who underwent EBUS-EUS staging during the study period. Twelve patients had EUS biopsy of station 6, and in 2 patients EUS biopsy was precluded by patient anatomy. One patient had an ectatic and enlarged aorta, precluding access to the station 6 lymph node. The other patient had heavy, irregular calcification on the aortic arch and left subclavian artery, and it was therefore considered too dangerous to pass the needle into the mediastinum without a clear path to the station 6 lymph node.

With EUS access, other mediastinal lymph nodes (aside from station 6) can successfully be biopsied even when as small as $2 \mathrm{~mm}$. EUS access of station 6 is more complicated and difficult because of the needle puncture of the lymph node very far from the esophageal puncture site and the movement of the aortic arch. The smallest lymph node biopsied in the current series was $0.8 \times 0.9 \mathrm{~cm}$. Smaller lymph nodes may be accessible; as the size of the lymph node decreases, however, the difficulty of the procedure increases.

The strengths of this study include the prospective nature of the data collection and complete follow-up in the entire cohort. Limitations of this study include the small number of patients and inherent selection bias. Two patients eligible for EUS biopsy of station 6 lymph nodes according to clinical and radiologic criteria were excluded from the series because of anatomic constraints. A further limitation includes the potential lack of generalizability as a result of the high volume EBUS-EUS practice of the thoracic endoscopists performing the procedures in this series.
In centers where EUS is available, this approach may become the first approach attempted for the biopsy of station 6 lymph nodes, reserving VATS or anterior mediastinotomy as second-line approaches. The EUS approach does not require general anesthesia in most cases; in addition, it is not associated with postprocedural pain and therefore does not necessitate analgesic medication on discharge. Furthermore, there are no wounds, thus obviating the risk of wound-related complications. The procedure can also be performed on an outpatient basis.

EUS access of para-aortic (station 6) lymph nodes allows complete, minimally invasive, endoscopic mediastinal lymph node staging and diagnosis without traversal of the aorta. By using a combined EBUS-EUS procedure, the thoracic endoscopist is capable of assessing all lymph node stations. The use of EUS to biopsy station 6 lymph nodes is safe and reliable for appropriately selected patients.

\section{References}

1. Coughlin M, Deslauriers J, Beaulieu M, Fournier B, Piraux M, Rouleau J, et al Role of mediastinoscopy in pretreatment staging of patients with primary lung cancer. Ann Thorac Surg. 1985;40:556-60.

2. Lemaire A, Nikolic I, Petersen T, Haney JC, Toloza EM, Harpole DH Jr, et al. Nine-year single center experience with cervical mediastinoscopy: complications and false negative rate. Ann Thorac Surg. 2006;82:1185-9; discussion 1189-90.

3. Vallières E, Pagè A, Verdant A. Ambulatory mediastinoscopy and anterior mediastinotomy. Ann Thorac Surg. 1991;52:1122-6.

4. Luke WP, Pearson FG, Todd TR, Patterson GA, Cooper JD. Prospective evaluation of mediastinoscopy for assessment of carcinoma of the lung. $J$ Thorac Cardiovasc Surg. 1986;91:53-6.

5. Nechala P, Graham AJ, McFadden SD, Grondin SC, Gelfand G. Retrospective analysis of the clinical performance of anterior mediastinotomy. Ann Thorac Surg. 2006;82:2004-9.

6. Wain JC. Video-assisted thoracoscopy and the staging of lung cancer. Ann Thorac Surg. 1993;56:776-8.

7. Naruke T, Asamura H, Kondo H, Tsuchiya R, Suemasu K. Thoracoscopy for staging of lung cancer. Ann Thorac Surg. 1993;56:661-3.

8. Roviaro G, Varoli F, Rebuffat C, Vergani C, Maciocco M, Scalambra SM, et al Videothoracoscopic staging and treatment of lung cancer. Ann Thorac Surg. 1995;59:971-4.

9. Wallace MB, Pascual JM, Raimondo M, Woodward TA, McComb BL, Crook JE, et al. Minimally invasive endoscopic staging in suspected lung cancer. JAMA 2008;299:540-6. 
10. Szlubowski A, Zieliński M, Soja J, Annema JT, Sośnicki W, Jakubiak M, et al. A combined approach of endobronchial and endoscopic ultrasound-guided needle aspiration in the radiologically normal mediastinum in non-small-cell lung cancer staging - a prospective trial. Eur J Cardiothoracic Surg. 2010;37:1175-9.

11. Mountain CF, Dresler CM. Regional lymph node classification for lung cancer staging. Chest. 1997;111:1718-23.

12. von Bartheld MB, Rabe KF, Annema JT. Transaortic EUS-guided FNA in the diagnosis of lung tumors and lymph nodes. Gastrointest Endosc. 2009;69:345-9.

13. Wallace MB, Woodward TA, Raimondo M, Al-Haddad M, Odell JA. Transaortic fine-needle aspiration of centrally located lung cancer under endoscopic ultrasound guidance: the final frontier. Ann Thorac Surg. 2007;84:1019-21.

14. Liberman M, Duranceau A, Grunenwald E, et al. New technique using EUS access for biopsy of para-aortic (station 6) mediastinal lymph nodes without traversing the aorta (with video). Gastrointestinal Endosc. 2011;73:1048-51.

15. Mountain CF. Revisions in the International System for Staging Lung Cancer. Chest. 1997;111:1710-7.

16. Silvestri GA, Tanoue LT, Margolis ML, Barker J, Detterbeck F. American College of Chest Physicians. The noninvasive staging of non-small cell lung cancer: the guidelines. Chest. 2003;123(1 Suppl):147S-56S

17. Cerfolio RJ, Bryant AS, Eloubeidi MA. Assessing the aortopulmonary window (\#5) and the paraaortic window (\#6) lymph nodes in patients with non-small cell lung cancer. Ann Thorac Surg. 2007;84:940-5.

18. Vilmann P, Puri R. The complete "medical" mediastinoscopy (EUS-FNA + EBUS-TBNA). Minerva Med. 2007;98:331-8.

19. Herth F, Lunn W, Eberhardt R, Becker HD, Ernst A. Transbronchial versus transesophageal aspiration of enlarged mediastinal lymph nodes. Am J Respir Crit Care Med. 2005;171:1164-7.

20. Rintoul RC, Skwarski KM, Murchison JT, Wallace WA, Walker WS, Penman ID. Endobronchial and endoscopic ultrasound-guided real-time fine-needle aspiration for mediastinal staging. Eur Respir J. 2005;25:416-21.

21. Annema JT, Rabe KF. State of the art lecture: EUS and EBUS in pulmonary medicine. Endoscopy. 2006;38(Suppl. 1):S118-22.

22. Annema JT, Versteegh MI, Veseliç M, Welker L, Mauad T, Sont JK, et al. Endoscopic ultrasound added to mediastinoscopy for preoperative staging of patients with lung cancer. JAMA. 2005;294:931-6.

23. Okamoto H, Watanabe K, Nagatomo A, et al. Endobronchial ultrasonography for mediastinal and hilar lymph node metastases of lung cancer. Chest. 2002;121: 1498-506.

\section{Discussion}

Dr Chumy Nwogu (Buffalo, $N Y$ ). This was a very fascinating presentation. I really enjoyed it. I have a question about how you were able to maneuver the needle after you got it out lateral to the arch. That seems technically difficult.

My second question is, when you are imaging with this particular linear EUS scope, it seems to me-and the illustration I think was very helpful - that you are higher than or proximal to the nodes. So when you are imaging, how well do you see these nodes that are further downstream from where your probe is?

Dr Liberman. Thank you. These are very good questions. I will start with the second question. We completely stage the mediastinum on EUS after our EBUS procedure, and we start in the belly moving upward. We evaluate the celiac access, the liver, and the adrenal gland, and we come back for the inferior ligament nodes. Then, when we get to the station 5 and then the station 6 nodes, we are looking at the node through the aorta, as I showed on the picture. Once we have seen the node, we have measured it, and we feel that it is accessible, then we pull the scope back about 4 to $5 \mathrm{~cm}$ above the aortic arch. At that time, we no longer see that node.

Obviously in the images I showed you, those are the bigger nodes, because it was easier to demonstrate in the presentation; however, a lot of the nodes in this study were smaller than that, and they would be even harder to see in the high mediastinum.

And going along the same lines to answer your first question, EUS, unlike EBUS, has the advantage of having a scope not only with 2 wheels but also with a tip deflector, where the needle itself is being bent without the scope being bent. You have a very long needle, as long as $8 \mathrm{~cm}$. You can pass that needle out into the mediastinum.

The way we do it is that once we have decided where that node is, we slowly pull the scope back. It depends on the patient anatomy, but it is usually about 20 to $25 \mathrm{~cm}$ from the incisors. And then we pass the needle out into the mediastinum. The mediastinal tissues, being rigid or somewhat rigid in the fat of the mediastinum, hold the needle in place, and as we advance the needle, we turn the big wheel of the scope back and we use the tip deflector to turn the needle forward, thus allowing us to get around the aorta.

The key is that once you have passed out into the mediastinum just medial to the left subclavian artery, the main risk is gone. The risk then only becomes the potential for puncture of the pleura and possible pneumothorax, which we have not seen. But getting out into the mediastinum outside of the vessels is the key, and once you are out there, you have much more play and it is much easier to do. Again, as you said, it is technically a more difficult node to biopsy than is a station 7 or 4 or something else.

Dr Nwogu. You are implying that when you actually pass the needle then you are not visualizing the node at that particular moment. Is that what you are saying?

Dr Liberman. Correct.

Dr Nwogu. You are using the mental image that you have from the earlier visualization?

Dr Liberman. Correct.

Dr Nwogu. That lack of direct visualization seems to increase the risk of the procedure a little bit.

Dr Liberman. Yes, it is a technically difficult thing to do. The station 6 node is not a node that needs to be biopsied in most situations, but in the cases that it needs to be done, you are avoiding a Chamberlain or a left VATS procedure. This endoscopic approach is done as an outpatient procedure. There is extremely low risk. We have not seen any complications in our series.

And if you should get a pneumothorax, first of all, you would see it right away on EUS; it would be easy to see. We have not had it happen yet in this type of procedure. And it really would not be the end of the world. Most pneumothoraces caused by EUS do not need to be treated.

Dr David J. Sugarbaker (Boston, Mass). This is a very interesting presentation, and I have 2 questions. The first is, what would you say the learning curve is on what appears to be, as you said, a more complex, technically demanding procedure?

Dr Liberman. Thank you. This is a very good question. This is not the first node that you want to biopsy when you are starting your EUS practice. We are doing a high volume, about 600 EUS cases a year for multiple years, and we have a large experience with EBUS. But I think that it is doable. A thoracic surgeon will be able to do this technique a lot better and with a lot more confidence and safety than, for example a gastroenterologist. I think that the anatomy is well known to this group, and the ease or the lack of stress in that mediastinal area for people in this room will be a lot less than for a nonthoracic surgeon. 
But although it is technically more difficult, I think that it is doable, and it is learnable. It is certainly not more difficult than a VATS lobectomy!

Dr Sugarbaker. The other question I had is just in looking at the positioning of the scope, have you ever thought about placing an ultrasound bronchoscope in along with the esophagoscope so that you could position that just across from the node and at that point actually visualize? I think many of us are a little concerned about passing the needle without seeing the lymph node. Have you ever thought about doing something like that?

Dr Liberman. It is a very good question. We have done that in other situations with multiple scopes, EBUS, EUS, in the esophagus during other procedures, especially in the animal laboratory. In this situation we have not used it, because we have not found that it would be important in our practice. But it definitely is something that would be an option for people who would be scared to find out where that node is.

But do not forget, when you are doing this procedure, it is not blind. You are following the left subclavian artery; you do see it, and sometimes with a bigger node you will see it at the same time as you are passing out into the mediastinum. Again, you have just pulled back 1 or $2 \mathrm{~cm}$; in general, you are watching the node, it is 2 seconds later, and you see the artery, which you know enters the aorta. The risk of the procedure I think is more the arterial puncture and the potential pseudoaneurysm formation or mediastinal bleeding. If you get out into the mediastinum and then you cannot find a node, just pull your needle back and do it again. It is a very small hole that you are making in the esophagus.

Dr Sugarbaker. I will just correct you that thoracic surgeons are never scared-but they may be concerned.

Dr Liberman. Thank you.

Dr Charles R. Bridges (Charlotte, NC). Given that in your abstract you presented only 12 patients, do you think it is a little premature to declare this procedure to be safe and reproducible?

Dr Liberman. That is a very good point. This is a very early experience with a new technique. I know that there have been people who have trained with us who have gone out and tried to do this technique, some with success and some without success. At least in my experience, and from what I have heard from others, there has been no associated morbidity, but it is too early to say, and at 12 cases I think you make a very good point. We cannot say that this is safe in the long term. 